June 18, 1996

LBNL-038974, UCB-PTH-96/26

WIS-96/24/June-PH

hep-th/9606112

\title{
D-Branes on Calabi-Yau Spaces and Their Mirrors
}

\author{
Hirosi Ooguri, Yaron $\mathrm{Oz}^{*}$ and Zheng Yin \\ Department of Physics, University of California at Berkeley \\ 366 Le Conte Hall, Berkeley, CA 94720-7300, U.S.A. \\ and \\ Theoretical Physics Group, Mail Stop 50A-5101 \\ Lawrence Berkeley National Laboratory, Berkeley, CA 94720, U.S.A.
}

\begin{abstract}
We study the boundary states of D-branes wrapped around supersymmetric cycles in a general Calabi-Yau manifold. In particular, we show how the geometric data on the cycles are encoded in the boundary states. As an application, we analyze how the mirror symmetry transforms D-branes, and we verify that it is consistent with the conjectured periodicity and the monodromy of the Ramond-Ramond field configuration on a Calabi-Yau manifold. This also enables us to study open string worldsheet instanton corrections and relate them to closed string instanton counting. The cases when the mirror symmetry is realized as T-duality are also discussed.
\end{abstract}

*Permanent address: Department of Particle Physics, Weizmann Institute of Science, 76100 Rehovot Israel. 


\section{Introduction}

D-branes in type II string theories have been identified as Ramond-Ramond charged BPS states [1]. In the presence of a D-brane, the boundary conditions for open strings are modified in such a way that Dirichlet boundary conditions are allowed in addition to the Neumann boundary conditions. The study of D-branes and its applications has been mainly restricted to the cases where the D-brane worldvolume is flat. In [2], a study of D-branes wrapped on curved spaces has been carried out in the long wavelength limit.

In this paper we will present a framework at the SCFT level for the study of D-branes on Calabi-Yau spaces. Perturbative string computations in the presence of a D-brane can be formulated by using a boundary state which describes how closed strings are emitted or absorbed on the D-brane worldvolume. In the case of the fully Neumann boundary condition near the flat background, the boundary state was constructed in [3]. Our main object of study is the boundary state for a D-brane wrapping on a non-trivial supersymmetric cycle in a Calabi-Yau space. In particular, we examine how the geometric data on the cycle are encoded in the boundary state.

The analysis of the boundary state will enable us to find the way mirror symmetry transforms D-brane configurations. It has been observed that, for a Calabi-Yau 3-fold $M$, the mirror symmetry not only maps the even cohomology of $M$ to the odd cohomology of its mirror $\widetilde{M}$, but it does so while respecting the integral structure of the cohomologies [4]. Based on this, it was conjectured by Aspinwall and Morrison [5] that the RamondRamond field on a Calabi-Yau space must have a certain periodicity reflecting this integral structure. This way, the mirror map can be extended to the Ramond-Ramond field configurations. We will verify that this conjecture is consistent with the mirror map between D-brane configurations.

The precise understanding of the mirror symmetry between D-branes enables us to study open string worldsheet instanton effects. We will find that the chiral primary part of the boundary states for 0, 2 and 3-cycles in a Calabi-Yau 3-fold does not receive instanton corrections while the instanton corrections for 4 and 6-cycles can be expressed in term of the closed string worldsheet instantons on the same manifold.

The paper is organized as follows: In section 2 we classify boundary conditions for $N=2$ SCFT which preserves half of the spacetime supersymmetry and the $N=1$ worldsheet supersymmetry. We then examine how these boundary conditions are realized by D-branes wrapping on cycles in a Calabi-Yau manifold. One may regard this as a microscopic version of the analysis by K. Becker, M. Becker and Strominger [6], where 
they studied the condition on the supersymmetric cycles using the low-energy effective actions for $p$-branes. In section 3 we will study the algebraic and geometrical structures of the boundary states of D-branes wrapped on supersymmetric cycles in Calabi-Yau spaces. We will distinguish between the middle-dimensional and even-dimensional cycles, and find the dependence of the boundary states on the choice of the cycles as well as the complex and Kähler moduli of the Calabi-Yau space. Section 4 will be devoted to the analysis of the mirror transformation of D-brane configurations. In section 5 we will present examples where the mirror symmetry is realized as T-duality on tori and Calabi-Yau orbifolds. Section 6 will be devoted to a discussion. In the appendix we discuss the construction of boundary states for Gepner models. We present an example that exhibits the relation between the boundary conditions for the model and the supersymmetric cycles.

\section{Supersymmetric cycles in Calabi-Yau manifolds}

In this section we will classify the boundary conditions for $N=2$ SCFT which preserves half of the spacetime supersymmetry and the $N=1$ worldsheet supersymmetry. We will then examine how these boundary conditions are realized by D-branes wrapping on cycles in a Calabi-Yau manifold. Here we will consider the case when the sigma-model for the Calabi-Yau manifold has one set of $N=2$ superconformal algebra for the leftmovers and one set for the right movers. It is straightforward to extend this analysis to the case where we have more than one set of $N=2$ algebras, such as $T^{2 d}$ with $d \geq 2$.

\subsection{Boundary conditions for $N=2$ SCFT}

The supersymmetric sigma-model for a Calabi-Yau manifolds has $N=2$ superconformal algebra (SCA). Throughout this paper, we set the signs of the left and the right $U(1)$ currents to be

$$
J_{L}=g_{i \bar{j}} \psi_{L}^{i} \psi_{L}^{\bar{j}}, \quad J_{R}=g_{i \bar{j}} \psi_{R}^{i} \psi_{R}^{\bar{j}},
$$

which determines the convention for $G^{ \pm}$as

$$
\begin{aligned}
& G_{L}^{+}=g_{i \bar{j}} \psi_{L}^{i} \partial X^{\bar{j}}, \quad G_{L}^{-}=g_{i \bar{j}} \psi_{L}^{\bar{j}} \partial X^{i}, \\
& G_{R}^{+}=g_{i \bar{j}} \psi_{R}^{i} \bar{\partial} X^{\bar{j}}, \quad G_{R}^{-}=g_{i \bar{j}} \psi_{R}^{\bar{j}} \bar{\partial} X^{i} .
\end{aligned}
$$

In addition, in order to preserve half of the spacetime supersymmetry, we should take into account the spectral flow operator $e^{i \phi_{L}}$ defined by

$$
e^{i \phi_{L}}=\Omega_{i_{1} \ldots i_{d}} \psi^{i_{1}} \ldots \psi^{i_{d}}
$$


Here $\Omega$ is the holomorphic $d$-form on the Calabi-Yau $d$-fold and $J_{L}=i \partial \phi_{L}$. Note that, in this convention, the $N=1$ supercurrent is generated by

$$
G=G_{L}^{+}+G_{L}^{-}
$$

In order to represent a BPS saturated state in spacetime, the boundary must preserve half of the spacetime supersymmetry. Thus we require the boundary state to be invariant under a linear combination of the left and right $N=2$ algebra extended by the spectral flow operators. Consistency restricts the linear combination to correspond to the automorphism group of the algebra. The automorphism is $O(2)$ for $N=2 \mathrm{SCA}$ and $Z_{2}$ for $N=1$. Since the supercurrent $G$ is gauged, its form should be preserved. Thus we are left with a $Z_{2} \times Z_{2}$-wise choice:

\section{A-type boundary condition:*}

$$
J_{L}=-J_{R}, \quad G_{L}^{+}= \pm G_{R}^{-}, \quad e^{i \phi_{L}}=e^{-i \phi_{R}}
$$

\section{B-type boundary condition:}

$$
J_{L}=+J_{R}, \quad G_{L}^{+}= \pm G_{R}^{+}, \quad e^{i \phi_{L}}=( \pm 1)^{d} e^{i \theta} e^{i \phi_{R}}
$$

The phase factor $e^{i \theta}$ will be determined later. In the A-type boundary condition, it can be absorbed in the definition of $\Omega$. This is why we did not put the phase factor in (2.5). Clearly both A-type and B-type boundary conditions preserve the $N=1 \mathrm{SCA}$

$$
T_{L}=T_{R}, \quad G_{L}= \pm G_{R},
$$

where $T$ denotes the stress tensor. It should be noted that the mirror symmetry exchanges the A-type and the B-type boundary conditions.

\section{$2.2 N=4$ SCFT}

In the case of string compactification on K3, the spectral flow operators have the conformal weight 1 . Combined with the $U(1)$ current $J$, they form the affine $S U(2)$ algebra and $N=2 \mathrm{SCA}$ is extended to $N=4$. For later convenience, let us write the holomorphic 2-form and the Kähler form as

$$
\Omega=k^{1}+i k^{2}, \quad k=k^{3} .
$$

\footnotetext{
*In this section we write boundary conditions in the notation appropriate for the open string channel.
} 
The $S U(2)$ currents are then

$$
J^{I}=k_{\mu \nu}^{I} \psi_{L}^{\mu} \psi_{L}^{\nu} \quad(I=1,2,3)
$$

where the indices $\mu, \nu$ refer to real coordinates on $\mathrm{K} 3$.

In addition to $G^{ \pm}$, we have two more supercurrents, which together with the original two form a $\underline{4}$ of $S O(4)$, the automorphism group of $N=4$ SCA. The automorphism consists of the internal and the external parts, $S U(2)_{c} \times S U(2)_{f}$, where $S U(2)_{c}$ is generated by the $S U(2)$ currents $J^{a}$ and $S U(2)_{f}$ is the external automorphism of the N=4 SCA [7]. We can then organize the four supercurrents as $(\underline{2}, \underline{2})$ of $S U(2)_{c} \times S U(2)_{f}$ as

$$
\begin{array}{ll}
G^{+-}=g_{i \bar{j}} \psi_{L}^{i} \partial X^{\bar{j}}, & G^{++}=\Omega_{i j} \psi_{L}^{i} \partial X^{j} \\
G^{-+} & =g_{i \bar{j}} \psi_{L}^{\bar{j}} \partial X^{i},
\end{array}
$$

In this notation, the $N=1$ supercurrent $G$ is

$$
G=G^{+-}+G^{-+}
$$

which is a singlet under the diagonal action of $S U(2)_{c} \times S U(2)_{f}$. Since $G$ is fixed, a general boundary condition which preserves both the $\mathrm{N}=4$ and $\mathrm{N}=1$ should only involve the diagonal subgroup of $S U(2)_{c} \times S U(2)_{f}$, i.e. $S O(3)$ in the full automorphism $S O(4)$. By decomposing the four supercurrents into $\underline{3}$ and $\underline{1}$ of $S O(3)$, the most general boundary condition is written as

$$
J_{L}^{I}=U_{J}^{I} J_{R}^{J}, \quad G_{L}^{I}= \pm U_{J}^{I} G_{R}^{J}, \quad G_{L}= \pm G_{R}, \quad(I, J=1,2,3)
$$

where $U \in S O(3)$.

\subsection{Geometric realization - general case}

We would like to find out how the above classification of supersymmetric boundary conditions corresponds to that of D-branes in a Calabi-Yau manifold $M$. In this section, we seek this identification in the large volume limit of $M$, where we can treat the sigmamodel semi-classically.

We begin by noting that (2.7) is solved by

$$
\partial X^{\mu}=R_{\nu}^{\mu} \bar{\partial} X^{\nu}, \quad \psi_{L}^{\mu}= \pm R_{\nu}^{\mu} \psi_{R}^{\nu}
$$

for some matrix $R$ provided it satisfies

$$
g_{\mu \nu} R_{\rho}^{\mu} R_{\sigma}^{\nu}=g_{\rho \sigma}
$$


The eigen-vector of $\mathrm{R}$ with eigen-value $(-1)$ gives the Dirichlet boundary condition for $X$, and thus should correspond to directions normal to the $\mathrm{D}$-brane. If the matrix $R$ is symmetric, the orthogonal directions are also eigenvectors of $R$ with eigen-values $(+1)$, and thus they obey the Neumann boundary condition corresponding to the tangential directions to the D-brane. In general, however, $R$ does not have to be symmetric, and this gives rise to a mixed Neumann-Dirichlet condition. As we will see, this corresponds to the case when the $U(1)$ gauge field on the D-brane worldvolume has non-zero field strength.

In the neighborhood of a $p$-cycle $\gamma$ on the Calabi-Yau $d$-fold, we can choose local coordinates such that $x^{A}(A=1, \ldots, p)$ are coordinates on the cycle and $y^{a}(a=1, \ldots, 2 d-$ $p)$ are for the directions normal to $\gamma$. Clearly $(2 d-p)$ is equal to the number of $(-1)$ eigen-values of $R$.

Suppose the D-brane wrapping on $\gamma$ gives the B-type boundary condition. It follows from (2.6) that $R$ should satisfy

$$
\begin{aligned}
k_{\mu \nu} R_{\rho}^{\mu} R_{\sigma}^{\nu} & =k_{\rho \sigma} \\
\Omega_{\mu_{1} \ldots \mu_{d}} R_{\nu_{1}}^{\mu_{1}} \ldots R_{\nu_{d}}^{\mu_{d}} & =e^{i \theta} \Omega_{\nu_{1} \ldots \nu_{d}} .
\end{aligned}
$$

The first of these equations implies

$$
k_{A b}=0,
$$

namely the Kähler form $k$ must be block diagonal on $\gamma$ in the tangential and the normal directions to $\gamma$. Since $k$ is nondegenerate, $k_{A B}$ and $k_{a b}$ must also be nondegenerate. This means the dimensions $p$ of the cycle must be even. Because $k$ is block diagonal, we can use it to define almost complex structure on the cycle. In fact it is integrable and defines a complex structure on the cycle. Thus $\gamma$ is a holomorphic submanifold of $M$. In the complex coordinates, the nonvanishing components of the top form $\Omega$ has $p / 2$ holomorphic indices tangential to $\gamma$ and $d-p / 2$ holomorphic indices normal to it. This determines the phase $e^{i \theta}$ in (2.15) in terms of the background gauge field on $\gamma$. In particular when the gauge field is flat, we find $e^{i \theta}=(-1)^{d-p / 2}$.

On the other hand, if the cycle corresponds to the A-type boundary condition, (2.5) implies

$$
\begin{aligned}
k_{\mu \nu} R_{\rho}^{\mu} R_{\sigma}^{\nu} & =-k_{\rho \sigma}, \\
\Omega_{\mu_{1} \ldots \mu_{d}} R_{\nu_{1}}^{\mu_{1}} \ldots R_{\nu_{d}}^{\mu_{d}} & =\bar{\Omega}_{\nu_{1} \ldots \nu_{d}} .
\end{aligned}
$$

If the background gauge field on $\gamma$ is flat, $R$ squares to the identity matrix. In this case, the first of the above equations implies

$$
k_{a b}=0, \quad k_{A B}=0 .
$$


Since $k$ is nondegenerate, this is possible only if $p=d$. Thus a cycle without a gauge field must be middle-dimensional. In this case, all the components of the holomorphic $d$-form $\Omega$ are related to $\Omega_{A_{1} \cdots A_{d}}$ as

$$
\Omega_{a_{1} \cdots a_{m} A_{m+1} \cdots A_{d}} \sim k_{a_{1}}^{A_{1}} \cdots k_{a_{m}}^{A_{m}} \Omega_{A_{1} \cdots A_{d}},
$$

for $m=1, \ldots, d$. Since $\Omega \wedge \bar{\Omega}$ is proportional to the volume form of the $d$-fold, it follows that the pull-back of $\Omega$ onto the cycle is proportional to its volume form. We note that the same geometric condition for supersymmetric cycles also arises from the low-energy effective worldvolume action of the supermembrane [6] in the case of $p=3$. It is easy to generalize this to the case with background gauge field. One can see that (2.17) implies $p=d, d+2, \ldots, 2 d$. The reason for this will become clear in the later sections.

\subsection{Geometric realization - K3 case}

In the case of K3, (2.12) states that $k^{I}(I=1,2,3)$ behave as

$$
k_{\mu \nu}^{I} R_{\rho}^{\mu} R_{\sigma}^{\nu}=U_{J}^{I} k_{\rho \sigma}^{J} .
$$

on the cycle $\gamma$. By going through some linear algebra, we find that the conjugacy class of the rotation $U$ is completely determined by the gauge field. For example, in the absence of the gauge field, the matrix $U$ is equal to 1 for 0 -cycle and 4-cycle while it is in the conjugacy class of $\pi$-rotation for 2-cycle. To understand this more geometrically, we diagonalize $U$ as

$$
U=M^{t}\left(\begin{array}{rrr}
\cos \theta & -\sin \theta & 0 \\
\sin \theta & \cos \theta & 0 \\
0 & 0 & 1
\end{array}\right) M
$$

By introducing a new basis by $M \in S O(3)$ rotation

$$
\tilde{k}^{I}=M_{J}^{I} k^{J}
$$

(2.20) is expressed as

$$
\begin{aligned}
\tilde{k}_{\mu \nu}^{3} R_{\rho}^{\mu} R_{\sigma}^{\nu} & =\tilde{k}_{\rho \sigma}, \\
\tilde{k}_{\mu \nu}^{ \pm} R_{\rho}^{\mu} R_{\sigma}^{\mu} & =e^{ \pm i \theta} \tilde{k}_{\rho \sigma}^{ \pm} .
\end{aligned}
$$

Comparing this with the analysis of the B-type boundary condition in the previous subsection, we see that the cycle $\gamma$ is a holomorphic submanifold of K3 with respect to the complex structure such that $\tilde{k}^{3}$ is a Kähler form and $\tilde{k}^{+}$is a holomorphic 2-form. Namely the $S O(3)$ rotation by $U$ reflects the $S O(3)$-wise choice of complex structure for a given

metric on K3. This result also agrees with the analysis in [6], [2]. 


\subsection{Summary}

We now summarize our classification of boundary conditions. For each complex dimension $d$ of the Calabi-Yau manifold, we designate allowed values of $p$ (real dimensions of the cycle) and their possible boundary conditions by type A, B or the one parameterized by $S O(3)$.

\begin{tabular}{||l|r|r|r|r|r|r|r|r|r|r|r|r|r|r|r|r||}
\hline$d$ & 1 & & & 2 & & & 3 & & & & & 4 & & & & \\
\hline$p$ & 0 & 1 & 2 & 0 & 2 & 4 & 0 & 2 & 3 & 4 & 6 & 0 & 2 & 4 & 6 & 8 \\
\hline Condition & $\mathrm{B}$ & $\mathrm{A}$ & $\mathrm{B}$ & $\mathrm{B}$ & $S O(3)$ & $\mathrm{B}$ & $\mathrm{B}$ & $\mathrm{B}$ & $\mathrm{A}$ & $\mathrm{B}$ & $\mathrm{B}$ & $\mathrm{B}$ & $\mathrm{B}$ & $\mathrm{A} / \mathrm{B}$ & $\mathrm{B}$ & $\mathrm{B}$ \\
\hline
\end{tabular}

This table is for the case with flat gauge field on $\gamma$. It is straightforward to generalize this to the case with non-zero gauge field strength.

One may notice that $p=3$ and 5 for $d=4$ are not included in the table*, even though there are Calabi-Yau 4 -folds with non-trivial $H_{3}$. From the above analysis it is clear that, provided the sigma-model for the 4-fold has only one set of $N=2$ SCA, one cannot construct a boundary condition at the SCFT level corresponding to a 3-cycle which preserves half of the spacetime supersymmetry. One arrives at the same conclusion by extending the analysis of [6] to the case when the membrane wraps around a 3-cycle in a 4 -fold. On a generic 4-fold with $S U(4)$ holonomy, there are two covariantly constant spinors $\epsilon_{1}$ and $\epsilon_{2}$ of the same chirality. One then finds that no linear combination of $\epsilon_{1}$ and $\epsilon_{2}$ can generate spacetime supersymmetry which preserves the membrane configuration. This does not mean that there is no Calabi-Yau 4-fold with a supersymmetric 3-cycle. To the contrary, one can construct orbifold examples which have such cycles. In these examples, however, the $N=2 \mathrm{SCA}$ is extended and thus the above classification is not applicable. Thus, an existence of a supersymmetric 3-cycle should imply an extension of the worldsheet $N=2 \mathrm{SCA}$. In general, a 4 -fold can have a holonomy group $\operatorname{Spin}(7)$, $S U(4), S p(2)$ or $S U(2) \times S U(2)$ [8]. The last two cases correspond, for instance, to the manifolds $T^{4} \times K 3$ and $K 3 \times K 3$ respectively, and the associated worldsheet algebras are extensions of the $N=2$ SCA. The generalization of the above classification of boundary conditions to these cases is straightforward. The analysis for the $\operatorname{Spin}(7)$ holonomy case will be reported elsewhere [9].

\footnotetext{
${ }^{*}$ We would like to thank C. Vafa for drawing our attention to this.
} 


\section{Boundary states for D-branes}

In this section, we examine the properties of the boundary states for D-branes wrapping on the supersymmetric cycles discussed in the previous section. We will show how the geometric data of the cycles are encoded in the boundary states.

\subsection{Supersymmetric boundary states}

Given the Virasoro algebra or its extension, there is a definite procedure for constructing a conformally invariant boundary state, where the left and right generators of the algebra are linearly related, starting from each highest weight state of the algebra. Denote by $|j, n\rangle, \overline{|j, n\rangle}$ orthonormal basis of the representations $j$ of the holomorphic and antiholomorphic algebras respectively. It has been shown by Ishibashi [10] that

$$
|j\rangle\rangle=\sum_{n}|j, n\rangle \otimes U \overline{|j, n\rangle}
$$

is such a state, where $U$ is an anti-unitary matrix which preserves the highest weight state $|j\rangle$. A boundary state is in general a linear combination of $|j\rangle\rangle$.

Type II strings compactified on Calabi-Yau spaces posses the worldsheet $N=2 \mathrm{SCA}$ in both the left and right sectors. As we saw in the previous section, a D-brane wrapping on a supersymmetric cycle preserves a linear combination of the left and right $N=2$ algebras. We would like to study the correspondence, D-branes $\leftrightarrow$ boundary states, for D-branes wrapped on supersymmetric cycles in Calabi-Yau spaces. In particular, given a D-brane, we would like to find the highest weight states that appear in its boundary state and their multiplicity, and conversely for a given boundary state we would like to find the D-brane configuration.

Recall from the analysis of section 2 that, for the closed strings, there are two types of supersymmetric boundary conditions: For middle-dimensional cycles, we have

$$
G_{L}^{+}= \pm i G_{R}^{-}, \quad G_{L}^{-}= \pm i G_{R}^{+}, \quad J_{L}=J_{R}
$$

and for even-dimensional cycles

$$
G_{L}^{+}= \pm i G_{R}^{+}, \quad G_{L}^{-}= \pm i G_{R}^{-}, \quad J_{L}=-J_{R}
$$

Here we are using the notation appropriate for the closed string channel* . They are called the A-type and the B-type boundary conditions. For the K3 case, the boundary conditions

\footnotetext{
${ }^{*} J_{R} \rightarrow-J_{R}$ and $G_{R}^{ \pm} \rightarrow i G_{R}^{ \pm}$compared to the notation in section 2.
} 
are parameterized by $S O(3)$ corresponding to the $S O(3)$-wise choice of complex structures for a given metric on K3. The boundary states realizing the A and B-type conditions should then satisfy

$$
\left(G_{L}^{+} \mp i G_{R}^{-}\right)|B\rangle=0, \quad\left(G_{L}^{-} \mp i G_{R}^{+}\right)|B\rangle=0, \quad\left(J_{L}-J_{R}\right)|B\rangle=0
$$

or

$$
\left(G_{L}^{+} \mp i G_{R}^{+}\right)|B\rangle=0, \quad\left(G_{L}^{-} \mp i G_{R}^{-}\right)|B\rangle=0, \quad\left(J_{L}+J_{R}\right)|B\rangle=0,
$$

depending on whether the boundary conditions are A-type or B-type. Let us examine the properties of these boundary states.

\subsection{A-type boundary condition}

Let us consider first the A-type boundary condition corresponding to middle- dimensional cycles. The boundary state can be expanded in terms of the Ishibashi states as

$$
\left.|B\rangle=\sum_{a} c^{a}|a\rangle\right\rangle
$$

where the sum is over the highest weight states of the $N=2$ algebra which appear in the Hilbert space of the sigma-model for the Calabi-Yau space $M$. They may be chiral primary states or non-chirals. According to our convention (2.2), complex moduli of $M$ are associated to $(c, c)$ and $(a, a)$ primary states and Kähler moduli are included in and $(c, a)$ and $(a, c)$.

The requirement that $\left(J_{L}-J_{R}\right)=0$ at the boundary implies $q_{L}=q_{R}$ for the $U(1)$ charges and thus a selection rule for the conformal fields that can contribute to the boundary state. In particular, this means that the coefficients in front of the $(c, a)$ and $(a, c)$ primaries are zero. In the following we will find an explicit form for the coefficients $c^{a}$ for the $(c, c)$ and $(a, a)$ chiral primary states.

For the sigma-model, the $(c, c)$ primaries with charge $(q, q)$ correspond to elements of the middle cohomology $H^{q, d-q}(M)$ where $d=\operatorname{dim}_{C} M$. It is straightforward to show that the coefficient $c^{a}$ corresponding to the $(c, c)$ primary state is given by

$$
c^{a}=\eta^{a b}\left\langle 0_{t o p}\left|\phi_{b}(z, \bar{z})\right| B\right\rangle_{\text {Ramond-Ramond }},
$$

where $\left\langle 0_{t o p}\right|$ is the topological vacuum of the A-model, $\eta^{a b}$ is the topological metric, and $\phi_{b}$ is the $(c, a)$ primary field associated to $\omega_{b} \in H^{q, d-q}(M)$. By the A-model, we mean the one with the topological twist such that $G_{L}^{+}$and $G_{R}^{-}$become one-forms on the worldsheet*.

${ }^{*}$ Thus the topological vacuum $\left\langle 0_{t o p}\right|$ has charges $(-d / 2,+d / 2)$. Since the $(c, a)$ primary field $\phi_{b}$ carries charges $(q, q-d)$, the total charges of $\left\langle 0_{t o p}\right| \phi_{b}$ is $(q-d / 2, q-d / 2)$ satisfying the selection rule. 
Since $\phi_{b}$ is physical in the A-model, and one may regard $c_{a}=\eta_{a b} c^{b}$ as a topological string amplitude on a disk with a puncture at $z$.

The coefficient $c_{a}$ may in principle depends on the Kähler moduli $\left(t^{i}, \bar{t}^{i}\right)\left(i=1, \ldots, h^{1,1}\right)$ as well as the complex moduli of $M$. To compute $\partial_{\bar{t}}$ of $c_{a}$, we insert $G_{L}^{+} G_{R}^{-} \bar{\varphi}_{i}$ onto the disk, where $\bar{\varphi}_{i}$ is an $(a, c)$ primary field with $\left(q_{L}, q_{R}\right)=(-1,1)$. Since both $G_{L}^{+}$and $G_{R}^{-}$are one-forms in the B-model, we can employ the standard contour deformation argument in the topological field theory. Taking into account the boundary condition $G_{L}^{+}= \pm i G_{R}^{-}$, one finds that the result of this insertion is zero. Thus $c_{a}$ is holomorphic in $t^{i}$ and therefore the instanton approximation to $c_{a}$ is exact.

Furthermore one can also show that $c_{a}$ is independent of $t^{i}$. One way to show this is to do the instanton expansion explicitly and verify that the instanton correction vanishes due to the fermion zero modes.

Another way to show this is to insert $G_{L}^{-} G_{R}^{+} \varphi_{i}$ where $\varphi_{i}$ is a $(c, a)$ primary field with $\left(q_{L}, q_{R}\right)=(-1,1)$. In this case, both $G_{L}^{-}$and $G_{R}^{+}$are two-forms on the disk and we cannot immediately deform their contours. On the disk with one puncture at $z$, there is a global holomorphic $(-1)$ form $\xi(w)=(w-z)(w-\bar{z})$. By multiplying $\xi$, we can convert $G_{L}^{-}$ into one-form and we can use the contour deformation argument. Since $\xi(w)$ vanishes at $w=z$, where $\phi_{a}$ is located, we can move the contour to the Dirichlet boundary where we can convert $\xi G_{L}^{-}$into $\bar{\xi} G_{R}^{+}$since $\xi$ is real-valued on the boundary (We chose the boundary to be $\operatorname{Im} w=0$.). We can them move $\bar{\xi} G_{R}^{+}$back and the contour slips out of the disk. Thus we have shown that $\partial_{t^{i}}$ of $c_{a}$ also vanishes. This reasoning is similar to the one which shows that the topological metric of the A-model does not receive the instanton correction.

Since $c_{a}$ is independent of the Kähler moduli, we can take the large volume limit in (3.7) to show

$$
c_{a}(\gamma)=\int_{\gamma} \omega_{a}
$$

where $\gamma$ is the supersymmetric cycle in question. Thus the chiral primary part of the boundary state is determined entirely by the homology class of the cycle $\gamma$.

This in particular means that the chiral primary part

$$
|\gamma\rangle=\sum_{\phi_{a}:(c, c)} c^{a}|a\rangle_{\text {Ramond-Ramond }}
$$

of the boundary state is a flat section of the so-called improved connection [11], [12], [13] for the bundle of Ramond vacua over the moduli space of $N=2$ superconformal field theories (for a review, see also section 2 of [14]). Since it plays an important role in the case 
of the B-type boundary condition in the following, let us demonstrate this fact explicitly here. Let us organize the basis of $H^{d}(M)$ as $\omega_{0} \in H^{d, 0}, \omega_{\alpha} \in H^{d-1,1}\left(\alpha=1, \ldots, h^{d-1,1}\right)$, etc. Then we find

$$
\frac{\partial c_{0}}{\partial \bar{y}^{\alpha}}=0, \quad \frac{D c_{0}}{D y^{\alpha}}=c_{\alpha}, \text { etc, }
$$

where $y^{\alpha}$ are the complex moduli of $M$ and $D$ is the covariant derivative on the vacuum line bundle $\mathcal{L}$ over the moduli space of the $N=2$ theories. These equations can be summarized as

$$
\nabla_{\alpha}|\gamma\rangle=0, \quad \bar{\nabla}_{\bar{\alpha}}|\gamma\rangle=0
$$

where

$$
\nabla_{\alpha}=D_{\alpha}-C_{\alpha}, \quad \bar{\nabla}_{\bar{\alpha}}=\bar{D}_{\bar{\alpha}}-\bar{C}_{\bar{\alpha}}
$$

and $C_{\alpha}$ is the multiplication by the Yukawa coupling.

This in particular means that $c_{a}$ for $\omega_{a} \in H^{d-1,1}$ etc, is obtained by acting with $D_{\alpha}$ on $c_{0}$. Thus the chiral primary part of the coefficients in (3.6) is completely determined by computing the period

$$
c_{0}(\gamma)=\int_{\gamma} \Omega
$$

of the holomorphic $(d, 0)$-form. To be precise, this is the case when the complex dimension of the Calabi-Yau manifold is less than 4 . When $d \geq 4$, there is some subtlety since there may be an element $\omega_{a}$ of $H^{d-q, q}$ with $q \geq 2$ which is not generated by differentiating $H^{d, 0}$ with respect to the complex moduli. If that is a case, we have to evaluate (3.8) for such $\omega_{a}$ separately. Understanding how this procedure works for $d \geq 4$ would help clarify issues on the mirror symmetry in higher dimensions [15].

\subsection{B-type boundary condition}

For an even-dimensional cycle $\tilde{\gamma}$, the boundary states satisfy the B-type condition $\left(J_{L}+J_{R}\right)|\tilde{B}\rangle=0$. Thus the coefficients $\tilde{c}^{a}$ for the expansion

$$
\left.|\tilde{B}\rangle=\sum_{a} \tilde{c}^{a}|a\rangle\right\rangle
$$

vanish for the $(c, c)$ and $(a, a)$ primary states. On the other hand, the coefficients for the $(c, a)$ primaries are obtained by

$$
\tilde{c}^{a}=\tilde{\eta}^{a b}\left\langle\tilde{0}_{t o p}\left|\tilde{\phi}_{b}(z, \bar{z})\right| \tilde{B}\right\rangle_{\text {Ramond-Ramond }}
$$

where $\left\langle\tilde{0}_{t o p}\right|$ is the topological vacuum of the B-model, $\tilde{\eta}^{a b}$ is the topological metric and $\tilde{\phi}_{a}(z, \bar{z})$ is the $(c, c)$ primary field associated to $\tilde{\omega}_{a}$ in the vertical series of the cohomologies 
$H^{\text {vertical }}(M)=\oplus_{q=0}^{d} H^{q, q}(M)$. The B-model is defined in such a way that $G_{L}^{+}$and $G_{R}^{+}$ behave as one-forms*.

By repeating the contour deformation argument as in the case of the A-type boundary condition, one finds that $c^{a}$ is independent of the complex moduli $y$, but may depend on the Kähler moduli $(t, \bar{t})$. We now present two arguments to show that the $(c, a)$ primary part of the boundary state

$$
|\tilde{\gamma}\rangle=\sum_{\tilde{\phi}_{a}:(c, a)} \tilde{c}^{a}|a\rangle_{\text {Ramond-Ramond }}
$$

is "flat" with respect to the improved connection over the Kähler moduli space. This determines the $(t, \bar{t})$ dependence of $\tilde{c}^{a}$.

A simple way to show this is to use the mirror symmetry. Since the mirror symmetry transforms the A-type boundary condition into the B-type, the flatness property of the state $|\gamma\rangle$ over the complex moduli space for the middle-dimensional cycle $\gamma$ should imply the flatness of $|\tilde{\gamma}\rangle$ over the Kähler moduli space for the even-dimensional cycle $\tilde{\gamma}$ provided $\gamma$ and $\tilde{\gamma}$ are related to each other by the mirror transform.

In the next section, we will use the flatness of $|\tilde{\gamma}\rangle$ to study the mirror symmetry between the D-branes. For the sake of completeness, we therefore give another argument for the flatness which stands independently of the mirror symmetry. To take a derivative of $\tilde{c}_{a}$ with respect to the Kähler moduli $t^{i}$, we insert $G_{L}^{-} G_{R}^{+} \varphi_{i}$ on the disk, where $\phi_{i}$ is a $(c, a)$ primary field corresponding to an element of $H^{1,1}$. Unlike the case of the complex moduli derivative, however, this does not yet give us $D_{i} \tilde{c}_{a}$ since $G_{L}^{-} G_{R}^{+} \varphi_{i}$ is divergent at the Dirichlet boundary. The covariant derivative $D_{i}$ must be defined in such a way that the contribution from the boundary is removed. Since $G_{R}^{+}$is a one-form in the $B$-model, we can deform its contour on the disk. By taking into account the boundary condition (3.5), one finds that $G_{L}^{-} G_{R}^{+} \varphi_{i}$ becomes $\partial \varphi_{i}$. The integral of $\partial \varphi_{i}$ over the disk with the puncture reduces to two surface integrals, one around the puncture at $z$ and another around the Dirichlet boundary. The former can be evaluated using the Yukawa coupling since it is related to the OPE of $H^{1,1}$ and $H^{q, q}$. The latter is canceled by the covariantization. This shows

$$
\left(D_{i}-C_{i}\right)|\tilde{\gamma}\rangle=0
$$

and similarly

$$
\left(\bar{D}_{\bar{i}}-\bar{C}_{\bar{i}}\right)|\tilde{\gamma}\rangle=0
$$

*Thus the topological vacuum $\left\langle\tilde{0}_{t o p}\right|$ has charges $(-d / 2,-d / 2)$ while $\tilde{\omega}_{a}$ carries $(d-q, q)$. Combined, they satisfy $q_{L}=-q_{R}$ as required. 
The flatness of $|\tilde{\gamma}\rangle$ implies that the coefficient $\tilde{c}_{0}$ corresponding to the top cohomology $H^{d, d}$ is holomorphic with respect to the Kähler moduli. It also implies that the rest of $\tilde{c}_{a}$ is obtained by taking derivatives of $\tilde{c}_{0}$ with respect to $t$. Since $\tilde{c}_{0}$ is holomorphic in $t$, the instanton approximation is exact, i.e. $\tilde{c}_{0}$ can be expressed as a sum over holomorphic maps from the disk to $M$ such that the boundary of the disc is mapped to the cycle $\tilde{\gamma}$. When $\tilde{\gamma}$ is $2 q$-dimensional, the contribution from the constant map can be evaluated by taking the large volume limit as

$$
\tilde{c}_{0}(\tilde{\gamma}) \sim \int_{\tilde{\gamma}} k^{q}+O\left(e^{2 \pi i t}\right)
$$

where $k=\sum_{i} t^{i} k_{i}$ and we choose $k_{i}$ to be the basis of $H^{1,1}(M ; Z)$.

The instanton corrections to $\tilde{c}_{0}$ are obtained by replacing the classical intersections in (3.19) by quantum ones in an appropriate sense. This in particular implies that $\tilde{c}_{0}$ for 0 or 2-cycle does not receive an instanton correction since the image of the holomorphic map of the disc does not intersect with the homology dual to $k_{i}$ in these cycles. In the next section, we will find that this in fact is consistent with the mirror symmetry.

The expressions (3.19) in particular means that the large volume limit of $\tilde{c}_{0}$ is a homogeneous polynomial of $t$ and the dimensions of the cycle $\tilde{\gamma}$ is characterized by the degree of the polynomial. One may be worried that this statement is not invariant under the integral shift of the theta parameters of the sigma-model, $t^{i} \rightarrow t^{i}+m^{i}\left(m^{i} \in Z\right)$. In fact this shift should mix cycles of different dimensions. Consider a cycle $\tilde{\gamma} \in H_{\text {vertical }}(M ; Z)$ and decompose it as

$$
\tilde{\gamma}=\sum_{q=0}^{d} \tilde{\gamma}_{q},
$$

where $\tilde{\gamma}_{q} \in H_{q, q}(M ; Z)$. The equation (3.19) can then be rewritten as

$$
\begin{aligned}
\tilde{c}_{0}(\tilde{\gamma}) & =\sum_{q} \int_{M} k^{q} \wedge \tilde{\gamma}_{q}^{*} \\
& =\int_{M} e^{k} \wedge\left(\sum_{q} q ! \tilde{\gamma}_{q}^{*}\right),
\end{aligned}
$$

where $\tilde{\gamma}_{q}^{*} \in H^{d-q, d-q}(M ; Z)$ is the Poincare dual of $\tilde{\gamma}_{q}$. One then finds that the shift $k \rightarrow k+\omega$ with $\omega \in H^{2}(M ; Z)$ mixes $\tilde{\gamma}_{q}$ 's as

$$
\tilde{\gamma}_{q}^{*} \rightarrow \sum_{n} q+n C_{n} \omega^{n} \wedge \tilde{\gamma}_{q+n}^{*}
$$

As we will see in the next section, this mixing is in accord with the mirror symmetry. 


\section{Mirror symmetry}

The mirror symmetry transforms type IIA string on a Calabi-Yau 3-fold $M$ into type IIB string on the mirror $\widetilde{M}$. Since type IIA string has even-dimensional D-branes while type IIB has odd-dimensional ones, we expect that the mirror symmetry to transform middle (=3) dimensional cycles on $M$ into even-dimensional cycle on $\widetilde{M}$. From the point of view of SCFT, the mirror symmetry transforms the A-type boundary condition (3.4) for the 3-cycle to the B-type boundary condition (3.5) for the even-dimensional cycle. In this section, we will examine how this transformation between the supersymmetric cycles takes place.

The mirror symmetry is an isomorphism between the Hilbert spaces of the sigmamodels on $M$ and $\widetilde{M}$ [16]. Thus if the cycles $\gamma$ and $\tilde{\gamma}$ are related to each other by the mirror symmetry, the corresponding boundary states $|B\rangle$ and $|\tilde{B}\rangle$ should be identified by the isomorphism*.

\subsection{Mirror map between cycles}

Suppose the boundary state $|B\rangle$ for a 3 -dimensional cycle $\gamma$ in $M$ is mapped to the boundary state $|\tilde{B}\rangle$ for an even-dimensional cycle $\tilde{\gamma}$ in $\widetilde{M}$ under the mirror transformation. Since the chiral primary part of the boundary states are characterized by $c_{0}$ and $\tilde{c}_{0}$ given in the previous section, they should be related to each other under the mirror map. For the 3 -cycle $\gamma, c_{0}$ is given by

$$
c_{0}(\gamma)=\int_{\gamma} \Omega .
$$

Since we know the large volume limit of $\tilde{c}_{0}$ as in (3.19), we should compare it with $c_{0}$ in the corresponding limit, which is called the large complex structure limit [17] of $M$.

In this limit, $H^{0,3}(M)$ aligns with the lattice of $H^{3}(M ; Z)$ [12], [4]. Thus we have a filtration of $H^{3}(M ; Z)$ in a form of

$$
H^{0,3} \subset H^{0,3} \oplus H^{1,2} \subset H^{0,3} \oplus H^{1,2} \oplus H^{2,1} \subset H^{3}(M ; Z),
$$

called the monodromy weight filtration [19]. Accordingly we can choose a symplectic basis $\left\{\alpha_{I}, \beta^{I}\right\}_{I=0, \ldots, h^{2,1}}$ for $H_{3}(M ; Z)$,

$$
\alpha_{I} \cap \alpha_{J}=0, \quad \beta^{I} \cap \beta^{J}=0, \quad \alpha_{I} \cap \beta^{J}=\delta_{I}^{J},
$$

* To be precise, the boundary state $|B\rangle$ does not belong to the Hilbert space since it is not normalizable. This problem can be easily avoided by considering $q^{L_{0}} \bar{q}^{\bar{L}_{0}}|B\rangle$ for $|q|<1$, for example. 
such that $\alpha_{0}$ is the unique cycle dual to $H^{0,3}$ and $\left\{\alpha_{0}, \ldots, \alpha_{h^{2,1}}\right\}$ spans the dual of $H^{0,3} \oplus$ $H^{1,2}$. The cycle $\alpha_{0}$ may also be characterized by the fact that it is invariant under the monodromy of $H_{3}(M ; Z)$ at the large complex structure limit [21], [23]. Note, on the other hand, $\alpha_{i}$ with $i=1, \ldots, h^{2,1}$ may be shifted by $\alpha_{0}$ under the monodromy transformation.

With this choice of the basis for $H_{3}$, the flat coordinates of the complex moduli space are given by

$$
s^{i}=\frac{X^{i}}{X^{0}} \quad\left(i=1, \ldots, h^{2,1}(M)=h^{1,1}(\widetilde{M})\right)
$$

where

$$
X^{0}=\int_{\alpha_{0}} \Omega, \quad X^{i}=\int_{\alpha_{i}} \Omega
$$

In the large complex structure limit $s \rightarrow \infty$ the Schmid orbit theorem [20] yields

$$
\begin{aligned}
& c_{0}\left(\beta^{0}\right)=\int_{\beta^{0}} \Omega=\frac{1}{3 !} X^{0} d_{i j k} s^{i} s^{j} s^{k}+\cdots, \\
& c_{0}\left(\beta^{i}\right)=\int_{\beta^{i}} \Omega=-\frac{1}{2 !} X^{0} d_{i j k} s^{j} s^{k}+\cdots,
\end{aligned}
$$

where $d_{i j k}$ is the large complex structure limit of the Yukawa coupling.

In order to construct the mirror map, we choose the standard gauge of the special geometry,

$$
c_{0}\left(\alpha_{0}\right)=\int_{\alpha_{0}} \Omega=1
$$

In this gauge, the flat coordinates are

$$
c_{0}\left(\alpha_{i}\right)=\int_{\alpha_{i}} \Omega=s^{i}
$$

By the mirror map, we may also use it as the flat coordinates for the Kähler moduli space of $\widetilde{M}$. In the large complex structure limit, this mirror symmetry maps the Yukawa coupling $d_{i j k}$ in (4.6) to

$$
d_{i j k}=\int_{\widetilde{M}} k_{i} \wedge k_{j} \wedge k_{k}
$$

By comparing large volume limit $(3.19)$ of $\tilde{c}_{0}$ for even-dimensional cycles in $\widetilde{M}$ with the large complex structure limit (4.6) - (4.9) of $c_{0}$ for $\left\{\alpha_{I}, \beta^{I}\right\}$, we can immediately see how the mirror symmetry transforms a D-brane wrapping on a 3-cycle in $M$ to a D-brane wrapping on an even-dimensional cycle in $\widetilde{M}$. In particular, the 3 -cycle $\alpha_{0}$ dual to $H^{0,3}$ in $M$ is a mirror image of a 0 -cycle in $\widetilde{M}$, and the 3 -cycles $\alpha_{i}\left(i=1, \ldots, h^{1,2}\right)$ correspond to 2-cycles in $\widetilde{M}$. Thus the mysterious correspondence between the integral structures of $H^{3}(M)$ and $H^{\text {vertical }}(\widetilde{M})$ pointed out in [4] is now understood as the mirror symmetry between the D-brane configurations. 
While this paper is being typed, we received a preprint [22] by Strominger, Yau and Zaslow where it is argued that the mirror of a 0-cycle in $\widetilde{M}$ should be a toroidal 3-cycle in $M$. Our analysis here shows a mirror of the 0 -cycle should be the 3 -cycle $\alpha_{0}$ dual to $H^{0,3}$ in the large complex structure limit of $M$. In the case of the quintic defined by,

$$
p(x)=x_{1}^{5}+x_{2}^{5}+x_{3}^{5}+x_{4}^{5}+x_{5}^{5}-5 \psi x_{1} x_{2} x_{3} x_{4} x_{5}=0,
$$

such a 3 -cycle is in fact known to be $T^{3}$ [18]. In the large complex structure limit $\psi \rightarrow \infty$, the holomorphic 3 -form becomes

$$
\Omega=5 \psi \frac{x_{5} d x_{1} \wedge d x_{2} \wedge d x_{3}}{\partial p / \partial x_{4}} \rightarrow-\frac{d x_{1} d x_{2} d x_{3}}{x_{1} x_{2} x_{3}},
$$

and the 3 -cycle dual to $\bar{\Omega}$ is $T^{3}$ surrounding $x_{1}=x_{2}=x_{3}=0$. It would be very interesting to see whether this feature of $H^{0,3}$ is true for a general $M$ with a mirror partner.

So far we have only looked at the large volume limit of $\widetilde{M}$ and the corresponding large complex structure limit of $M$. Fortunately, since the state $|\tilde{\gamma}\rangle$ and $|\tilde{\gamma}\rangle$ are flat sections over the moduli spaces, their correspondence can be traced to interiors on the moduli spaces following the mirror map. We will demonstrate this through examples in section 5. If we go around a non-trivial cycle over the moduli space, we have to deal with the monodromy problem, which we will discuss below.

\subsection{Open string instantons}

For the A-type boundary condition, the classical formula

$$
c_{0}(\gamma)=\int_{\gamma} \Omega
$$

is exact. On the other hand, the formula (3.19) for $\tilde{c}_{0}(\tilde{\gamma})$ for the B-type boundary condition is corrected by open string worldsheet instantons, i.e. holomorphic maps from a disk to $\widetilde{M}$ such that the boundary of the disk is mapped to the cycle $\tilde{\gamma}$. The mirror symmetry suggests that such open string instanton effects are expressed in terms of the closed string instantons on $\widetilde{M}$.

The mirror symmetry gives another proof for the fact that the formula (3.19) for $\tilde{c}_{0}$ does not receive the instanton correction when the cycle $\tilde{\gamma}$ is 0 or 2-dimensional. This is because the corresponding formulae (4.7) and (4.8) for $\alpha_{I}\left(I=0, \ldots, h^{2,1}\right)$ are, by definition, exact.

On the other hand, $\tilde{c}_{0}$ for 4 or 6 -cycle does receive instanton corrections. In the mirror picture, the exact formulae for $c_{0}(\gamma)$ for $\beta^{I}\left(I=0, \ldots, h^{2,1}\right)$ can be written in terms of the 
prepotential $\mathcal{F}$ for $M$ as

$$
\begin{array}{r}
c_{0}\left(\beta^{0}\right)=2 \mathcal{F}-s^{i} \frac{\partial}{\partial s^{i}} \mathcal{F}, \\
c_{0}\left(\beta^{i}\right)=\frac{\partial}{\partial s^{i}} \mathcal{F},
\end{array}
$$

where we are working in the $X^{0}=1$ gauge appropriate for the mirror symmetry. In $\widetilde{M}$, the prepotential is related to the sum over closed string instantons as*

$$
-\frac{\partial^{3}}{\partial s^{i} \partial s^{j} \partial s^{k}} \mathcal{F}=d_{i j k}+\sum_{n} N(n) n_{i} n_{j} n_{k} \frac{e^{2 \pi i n_{i} s^{i}}}{1-e^{2 \pi i n_{i} s^{i}}},
$$

where $N(n)$ is the number of rational curves on $\widetilde{M}$ of the type $n=\left\{n_{1}, \ldots, n_{h^{1,1}(\widetilde{M})}\right\}$. By integrating this, we find

$$
\mathcal{F}=-\frac{1}{3 !} d_{i j k} s^{i} s^{j} s^{k}+a-\sum_{n} \sum_{m=1}^{\infty} \frac{N(n)}{(2 \pi i m)^{3}} e^{2 \pi i m n_{i} s^{i}}
$$

where $a$ is a constant, presumably related to the four-loop term in the $\beta$-function of the sigma-model [18]. Substituting this into (4.13), we can extract the open string instanton corrections to $\tilde{c}_{0}$ and express them in terms of of the number of the closed string instantons $N(n)$.

This suggests a relation between the moduli spaces of open and closed string instantons and the corresponding intersection theories. One way to find such a relation may be to regard a closed string instanton intersecting a supersymmetric cycle as a pair of open string instantons glued on the cycle.

\subsection{Integral structure and monodromy}

It has been observed that in the large radius limit, the mirror symmetry maps the integer valued homology $H_{3}(M ; Z)$ to $\oplus_{q} H_{2 q}(\widetilde{M} ; Z)$ in such a way that the monodromy is preserved [4], [21]. Based on this, it was conjectured by Aspinwall and Morrison [5, 23] that the Ramond-Ramond fields on the Calabi-Yau 3-fold should have periodicity under the discrete shift reflecting these integral structures. This would guarantee that the mirror symmetry can be extended to the Ramond-Ramond fields configurations. This periodicity should be a consequence of the coupling of the Ramond-Ramond field to the worldvolume of the D-brane. In fact the mirror map between the D-branes we found in the above is consistent with this picture.

\footnotetext{
${ }^{*}$ We are using the same coordinates $s^{i}$ for both the complex moduli of $M$ and the Kähler moduli of $\widetilde{M}$ related to each other by the mirror symmetry.
} 
By requiring that the monodromy be preserved, Morrison also pointed out [23] that the shift of the NS-NS $B$-field by $H^{2}(M ; Z)$ should cause a certain rearrangement of the integral structure of the Ramond-Ramond fields of even ranks. This is also consistent with the mixing of the even dimensional cycles we found in (3.22).

Although the mixing of the cycles is required by the mirror symmetry, one can also explain it without invoking the mirror. For the sigma-model without a boundary, the shift of $B$-field by $H^{2}(M ; Z)$ is a discrete symmetry. However, in the presence of a boundary, the coupling of the $B$-field to the string world-sheet is accompanied by the coupling of a $U(1)$ gauge field $A$ to the boundary [24]. Since the gauge invariant field strength is $\mathcal{F}=F-B$ where $F=d A$, the shift $B \rightarrow B+\omega$ with $\omega \in H^{2}(M ; Z)$ is compensated by $F \rightarrow F+\omega$. This effectively mixes cycles of different dimensions as in (3.22). Below we will demonstrate this explicitly through examples.

\section{$5 \quad$ Examples}

In this section we will present several examples to illustrate the general results of the previous sections. We will show explicitly how starting with a D-brane wrapped on a middle-dimensional supersymmetric cycle, depending on the D-brane configuration and T-duality or mirror transformation, we can obtain different dimensionalities for the dual configuration with gauge fields background.

\section{$5.1 \quad$ T-duality on tori}

Let us start with a general discussion of the duality map for tori and orbifolds. As we discussed in section 2, the condition for $N=1$ SCA yields

$$
\partial X^{\mu}=R_{\nu}^{\mu} \bar{\partial} X^{\nu},
$$

where $R$ is an orthogonal matrix. The requirement for having a geometrical interpretation of a D-brane without gauge fields background is more restrictive and implies that $R$ has to be a symmetric matrix and squares to the identity matrix. In this case, its eigen-values are $(+1)$ or $(-1)$ corresponding to the tangential and normal vectors to the D-brane respectively. To preserve the $N=2 \mathrm{SCA}, R$ should further obey

$$
k_{\mu \nu} R_{\rho}^{\mu} R_{\sigma}^{\nu}= \pm k_{\rho \sigma},
$$

where \pm refers to the A and B-type boundary conditions and thus to middle and evendimensional cycles. 
T-duality transformation is realized by

$$
\partial X^{\mu} \rightarrow \partial X^{\mu}, \quad \bar{\partial} X^{\mu} \rightarrow T_{\nu}^{\mu} \bar{\partial} X^{\nu}
$$

where $T$ is the symmetric matrix implementing the duality transformation and $T^{2}=1$. In order for this to induce the mirror transformation, the sign of $J_{R}$ should be reversed while $J_{L}$ remain invariant. This means

$$
k_{\mu \nu} T_{\rho}^{\mu} T_{\sigma}^{\nu}=-k_{\rho \sigma} .
$$

Thus, starting with a D-brane configuration and performing T-duality transformation we will end up with a configuration satisfying the boundary condition

$$
\partial X^{\mu}=\tilde{R}_{\nu}^{\mu} \bar{\partial} X^{\nu}
$$

where $\tilde{R}=R T$ is an orthogonal matrix. If the matrix $\tilde{R}$ is symmetric and thus squares to the identity matrix, the boundary condition has geometrical realization as a D-brane without the $U(1)$ gauge field. This occurs if and only if

$$
[R, T]=0
$$

namely T-duality transformation commutes with the original D-brane configuration.

When (5.6) is not satisfied, we get a mixing between the Neumann and Dirichlet boundary conditions of the type induced by a background gauge field. Since $\tilde{R}=R T$ is orthogonal, by a coordinate transformation, we can alway bring it into the standard form,

$$
\tilde{R}=\left(\begin{array}{cc}
-1_{(2 d-p) \times(2 d-p)} & 0 \\
0 & \left(\frac{1-F}{1+F}\right)_{p \times p}
\end{array}\right),
$$

where for some $p$ and an anti-symmetric matrix $F$. This implies the Dirichlet boundary condition for the first $(2 d-p)$ directions, while the boundary condition for the second $p$ directions is

$$
\partial X^{\mu}=\left(\frac{1-F}{1+F}\right)_{\nu}^{\mu} \bar{\partial} X^{\nu}
$$

Therefore the matrix $\tilde{R}$ describes a $p$-cycle with a background gauge field $F$.

Whether $F$ is zero or not, the mirror symmetry exchanges odd and even-dimensional cycles when $d=\operatorname{dim}_{C} M$ is odd. In this case, the condition (5.4) for T-duality to be the mirror symmetry implies $\operatorname{det} T=-1$. On the other hand, $\operatorname{det} R=-1$ for an odd dimensional cycle since the rotation matrix $\left(\frac{1-F}{1+F}\right)$ has determinant $(+1)$. Thus $\tilde{R}=R T$ for its mirror obeys $\operatorname{det} \tilde{R}=\operatorname{det} R \cdot \operatorname{det} T=+1$, i.e. the mirror of the odd dimensional 
cycle is even-dimensional. If $R$ and $T$ commute, $F=0$ in the original cycle implies $F=0$ for its mirror.

Let us construct now a simple example to illustrate the above. Consider the torus $T^{2}$ with real coordinates $(x, y)$, and a D-brane configuration defined by the Pauli matrix $R=\sigma_{1}$. The Neumann boundary condition is imposed on the 1-cycle defined by the vector $(1,1)$, while the Dirichlet boundary condition is imposed on the vector orthogonal to it. Then the mirror transformation is generated by T-duality transformation along the $x$ coordinate, i.e. $T=-\sigma_{3}$. Clearly this $T$ does not commute with $R$. In fact $\tilde{R}=-i \sigma_{2}=F$, and this has no $(-1)$ eigen-value, namely there are no Dirichlet boundary conditions. The configuration we got is that of a 2-cycle with background gauge field $F$.

It is instructive to consider this example from a different viewpoint. In the limit of the large complex structure, $\tau \rightarrow i \infty$, the cohomology $H^{0,1}$ generated by $d \bar{z}=d x+\bar{\tau} d y$ gets aligned with the lattice $H^{1}\left(T^{2} ; Z\right)$ generated by $d x$ and $d y$. In this limit, the cycle $(1,0)$ becomes dual to $H^{0,1}$ and the mirror map transforms it to a 0 -cycle, as expected. On the other hand, either $(0,1)$ or $(1,1)$ can be combined with $(1,0)$ to make the symplectic basis of $H^{1}\left(T^{2} ; Z\right)$. Since $(0,1)$ is mirror to a 2 -cycle without a gauge field, one may regard $(1,1)=(0,1)+(1,0)$ as mirror to the 2 -cycle with a 0 -cycle on it. Though the filtration $H^{0,1} \subset H^{1}\left(T^{2} ; Z\right)$ makes sense only in the large complex structure limit, the mirror map between the cycles holds even for finite value of $\tau$. The reason for this can be traced back to the fact that the chiral primary part of the boundary state $|\gamma\rangle$ is a flat section over the moduli space of complex structure, as we explained in section 4 .

This picture is correct as far as the homology goes, but a sum of the straight lines, $(0,0) \rightarrow(1,0)$ and $(1,0) \rightarrow(1,1)$, is not actually supersymmetric since the combined cycle is not minimal. The diagonal line $(0,0) \rightarrow(1,1)$ is shorter and thus costs less energy. In the mirror picture, this means that the 2-cycle with the $U(1)$ gauge field should be regarded as a ground state of the 0 -cycle on the 2-cycles.

This simple example illustrates the mixing of cycles (3.22). The D-brane worldvolume action has terms of the form [25]

$$
S=\int_{2-\text { cycle }} C_{0} \mathcal{F}+C_{2},
$$

where $C_{0}$ and $C_{2}$ are the Ramond-Ramond fields and $\mathcal{F}=F-B$. A shift of $B$ by $H^{2}\left(T^{2} ; Z\right)$ then mixes $C_{0}$ and $C_{2}$ corresponding to the mixing of cycles. In the mirror picture, the shift $B \rightarrow B+1$ becomes the modular transformation $\tau \rightarrow \tau+1$. This sends the cycle $(0,1)$ (the 2-cycle in the mirror) to $(1,1)$ (the 0 -cycle on the 2-cycle in the mirror). Thus the mixing of the cycle (3.22) is natural from the point of view of the coupling of the D-brane to the $B$ field [24] as well as the mirror symmetry. 


\subsection{Calabi-Yau orbifold}

In this section we discuss an example of a mirror pair of Calabi-Yau orbifolds. In fact the phenomena is basically similar to the tori cases, with some technicality related to the correct choice of a ground state. As an explicit example we will consider the mirror of the Calabi-Yau orbifold $\left(T^{2}\right)^{3} /\left(Z_{2} \times Z_{2}\right)$ which is constructed by the inclusion of a discrete torsion [26]. Let us first discuss the orbifold without a discrete torsion. The Calabi-Yau orbifold $\left(T^{2}\right)^{3} / \Gamma$ where $\Gamma=Z_{2} \times Z_{2}$ is defined by $z_{i} \rightarrow(-1)^{\varepsilon_{i}} z_{i}, \quad i=1,2,3$ such that $\prod_{i}(-1)^{\varepsilon_{i}}=1$. Supersymmetric 2 cycles can constructed by projecting a $T^{2}$ in $\left(T^{2}\right)^{3}$ with respect to $\Gamma$. Similarly, supersymmetric 4-cycles can be obtained by projecting a product of two $T^{2}$ 's with respect to $\Gamma$. The even-dimensional supersymmetric cycles are interesting in this example since the twisted Ramond ground states contribute to $H^{1,1}$ and $H^{2,2}$. Thus the latter can show up in their boundary states.

Consider, for instance, a 2-cycle boundary state where Neumann boundary conditions are imposed on the $z_{3}$ coordinate and Dirichlet boundary conditions on $z_{1}, z_{2}$. Orbifold boundary states are simply constructed as a sum of contributions from the untwisted and twisted sectors

$$
|B\rangle_{\text {orbifold }}=|B\rangle_{\text {untwisted }}+\sum_{\text {twisted sectors }}|B\rangle_{\text {twisted }}
$$

with an appropriate projection on invariant states.

\section{untwisted sector:}

The boundary state takes the form

$$
|B\rangle_{\text {untwist }}=\exp \left(-\sum_{n=1}^{\infty} \frac{1}{n}\left(\sum_{i=1}^{2} \alpha_{L,-n}^{i} \alpha_{R,-n}^{i}+\alpha_{L,-n}^{* 3} \alpha_{R,-n}^{3}\right)+\text { c.c }\right)|0\rangle,
$$

and projection is not required since the boundary state is $\Gamma$-invariant. The fermionic part works similarly.

\section{twisted sectors:}

There exist three twisted sectors corresponding to the three $\Gamma$ group elements. Consider, for instance, the twisted sector corresponding to the generator $\alpha, \alpha\left(z_{1}, z_{2}, z_{3}\right)=$ $\left(-z_{1},-z_{2}, z_{3}\right)$, where the $\beta$ and $\gamma$ are defined by a permutation of the signs. This implies half integer modding for the first two coordinates and integer modding for the third. The other twisted sectors are simply permutations of that.

Let us consider now the inclusion of a discrete torsion. This simply amounts to a change in the projection operators in the twisted sectors. Thus in the sector twisted by $\alpha$ it amounts to an inclusion of another minus sign in the transformation of states under 
$z_{3} \rightarrow-z_{3}$. This has the effect that only twisted Ramond ground states that contribute to $H^{1,2}$ and $H^{2,1}$ survive the projection. Thus we end up with a Hodge diamond mirror to that of the orbifold without discrete torsion. It was argued in [26] that these indeed constitute a mirror pair, where the mirror map is T-duality.

Upon inclusion of a discrete torsion, the interesting supersymmetric cycles are the middle dimensional ones. The construction of a boundary state is standard and we can follow the duality map. There is, however, a delicate point. The discrete torsion changes the projection operator, and for instance in the $\alpha$ twisted sector it takes the form

$$
P=\frac{1}{4}(1+\alpha-\beta-\gamma)
$$

which naively annihilates the twisted sector boundary state. This is resolved by picking the correct ground state. Consider the Ramond sector: Related to $z_{3}$ we have the fermionic zero modes $\psi_{L, 0}^{3}, \psi_{R, 0}^{3}$ with the boundary condition

$$
\left(\psi_{L, 0}^{3}+i \eta \psi_{R, 0}^{3 *}\right)|0\rangle=0, \quad\left(\psi_{L, 0}^{3 *}+i \eta \psi_{R, 0}^{3}\right)|0\rangle=0
$$

with $\eta= \pm 1$.

Of the possible Ramond ground states only $\left(i \eta \psi_{L, 0}^{3}+\psi_{R, 0}^{3}+\right.$ c.c $)|0\rangle$ survives the projection and should be picked. This is to be contrasted with the case without discrete torsion where the correct twisted sector Ramond ground state is $\left(i \eta+\psi_{L, 0}^{3} \psi_{R, 0}^{3}+\right.$ c.c $)|0\rangle$.

Consider now the D-brane matrix $R=\operatorname{diag}\left[\sigma_{1}, \sigma_{1}, \sigma_{1}\right]$. A mirror symmetry transformation is defined by:

$$
\partial z_{i} \rightarrow \partial z_{i}, \quad \bar{\partial} z_{i} \rightarrow \bar{\partial} \bar{z}_{i}
$$

Thus the matrix $T$ takes the form $T=\operatorname{diag}\left[\sigma_{3}, \sigma_{3}, \sigma_{3}\right]$ and does not commute with $R$. Since both $R$ and the mirror symmetry $T$ are equivariant with respect to the $Z_{2} \times Z_{2}$ discrete group, the same applies for the Calabi-Yau orbifold $\left(T^{2}\right)^{3} /\left(Z_{2} \times Z_{2}\right)$, and we get the mixing phenomena as we discussed before.

In the orbifold models, we may consider gauge field strength which belongs to the twisted sectors, namely localized on a particular fixed point. In this case we should expect that the particular twisted sector corresponding to this fixed point will be influenced. Thus, we are led to consider different boundary conditions $R$ in (5.1) for the untwisted and twisted sectors. It would be interesting to further explore this structure. 


\section{Discussion}

We have shown that boundary states provide a framework at the SCFT level to study configurations of D-branes, wrapped on supersymmetric cycles in Calabi-Yau spaces, with implications on the structure of mirror symmetry between D-branes. There are various directions for future research.

Understanding the role of the non-chiral primary states in the boundary state is an important and challenging problem. This is important since the non-chiral part carries information on the moduli of supersymmetric cycles. One way to explore this issue is to use non-linear recursion relations for the boundary states which can be derived by moving vertex operators on the disc to its boundary and study the boundary states associated with the disc splitting.

Boundary states can be used in order to explore the moduli spaces of D-branes wrapped on supersymmetric cycles in Calabi-Yau spaces. This will have various applications such as D-brane states counting $[2,27]$, and may provide us with means to probe the structure of mirror symmetry as suggested in [22]. Moreover, we expect the boundary states to be also helpful in exploring mirror symmetry in higher dimensions.

The relation that we found between open string worldsheet instanton corrections and closed string instantons counting, points to a deep structure between the corresponding moduli spaces which should be revealed.

It has been shown in [28] that supersymmetric gauge theories can be realized via wrapping D-branes on supersymmetric cycles. The SCFT framework that we presented is likely to be useful in exploring this direction. 


\section{Acknowledgements}

We would like to thank K. Becker, T. Eguchi, D. Gepner, B. Greene, S. Katz, M. Li, J. Maldacena, D. Morrison, R. Plesser, J. Polchinski, A. Schwimmer, A. Strominger and C. Vafa for useful discussions. Y.O. would like to thank LBNL for hospitality during the final stages of this work. This work was supported in part by the National Science Foundation under grants PHS-9501018 and PHY-951497 and in part by the Director, Office of Energy Research, Office of High Energy and Nuclear Physics of the U.S. Department of Energy under Contract DE-AC03-76SF00098. Y.O. is partially supported by the Israel Science Foundation through the Center for the Physics of Basic Interactions. Z.Y. is supported by Graduate Research Fellowship of the U.S. Department of Education. 


\section{Appendix}

\section{A Boundary states for Gepner models}

A Gepner model [29] can be viewed as an orbifold construction in which we project out states that do no satisfy the required conditions and add twisted sectors to the Hilbert space. This suggests that the way to construct the boundary state for a Gepner model is to take the product of the boundary states for the minimal model parts with the appropriate projection and addition of twisted sectors.

In the following we consider the simplest example: The $(k=1)^{3}$ Gepner model. This corresponds to a sigma-model on $T^{2}$ with $Z_{3}$ symmetry. In this case, each minimal model can be constructed by a free boson. Thus we have $\phi_{i}, i=1,2,3$. Let us construct the boundary state for a D-brane wrapped on a supersymmetric 1-cycle in $T^{2}$. Imposing the A-type boundary conditions implies

$$
\phi_{L}^{i}=\phi_{R}^{i}+c_{i}
$$

with constants $c_{i}$

$$
c_{i}=\frac{2 \pi}{\sqrt{3}} n_{i}+\left(0 \text { or } \frac{2 \pi}{2 \sqrt{3}}\right),
$$

where $n_{i}$ are integers and the choice of 0 or $\frac{2 \pi}{2 \sqrt{3}}$ corresponds to the sign of the RamondRamond charge (i.e. BPS or anti-BPS). For each choice of $c_{i}$, the boundary state is uniquely constructed by the standard oscillator procedure.

It is instructive to interpret this from the sigma-model viewpoint. The sigma-model for $T^{2}$ consists of complex free boson $X$ and a complex free fermion $\psi$ which are related to $\phi_{i}$ by

$$
\begin{aligned}
\psi & =\exp \left[\frac{i}{\sqrt{3}}\left(\phi_{1}+\phi_{2}+\phi_{3}\right)\right] \\
\partial X & \left.=\exp \left[\frac{i}{\sqrt{3}}\left(-2 \phi_{1}+\phi_{2}+\phi_{3}\right)\right]+\text { (permutations in } 1,2,3\right) .
\end{aligned}
$$

The boundary conditions (A.1),(A.2) correspond in the sigma model to

$$
\begin{aligned}
\psi_{L} & = \pm e^{\frac{2 \pi i}{3}\left(n_{1}+n_{2}+n_{3}\right)} \psi_{R}, \\
\partial X & =e^{\frac{2 \pi i}{3}\left(n_{1}+n_{2}+n_{3}\right)} \bar{\partial} X .
\end{aligned}
$$

The case $n_{1}+n_{2}+n_{3}=0 \bmod 3$ corresponds to the Neumann boundary condition on the $\{X=$ real $\}$ cycle of $T^{2}$, while $n_{1}+n_{2}+n_{3}=1$ or $2 \bmod 3$ correspond to 
Neumann boundary conditions on the $Z_{3}$ related 1-cycles. We see that the different choices of boundary conditions for the Gepner model correspond to the different choices of supersymmetric 1-cycles. We expect that such relations between the algebraic and the geometric structures should exist in general.

The boundary state takes the form $|B\rangle=|B\rangle_{X}|B\rangle_{\psi}$ where

$$
\begin{aligned}
|B\rangle_{X} & =\exp \left[-e^{\frac{2 \pi i}{3}\left(n_{1}+n_{2}+n_{3}\right)}\left(\sum_{n=1}^{\infty} \frac{1}{n} \alpha_{L,-n} \alpha_{R,-n}+\text { c.c }\right)\right]|0\rangle, \\
|B\rangle_{\psi} & =\exp \left[ \pm i e^{\frac{2 \pi i}{3}\left(n_{1}+n_{2}+n_{3}\right)}\left(\sum_{n} \psi_{L,-n} \psi_{R,-n}+\text { c.c }\right)\right]|0\rangle .
\end{aligned}
$$

Note that from the chiral primary states only the $(c, c)$ ring $\left\{1, \psi_{L} \psi_{R}\right\}$ and its complex conjugate $(a, a)$ ring contribute to the boundary state as expected. 


\section{References}

[1] J. Polchinski, "Dirichlet-Branes and Ramond-Ramond Charges," hep-th 9510017, Phys. Rev. Lett. 75 (1995) 4724.

[2] M. Bershadsky, V. Sadov and C. Vafa, "D-Branes and Topological Field Theories," hep-th 9511222, Nucl. Phys. B463 (1996) 420.

[3] J. Polchinski and Y. Cai, "Consistency of Open Superstring Theories," Nucl. Phys. B296 (1988) 91; C. G. Callan, C. Lovelace, C. R. Nappi and S. A. Yost, "Loop Corrections to Superstring Equations of Motion," Nucl. Phys. B308 (1988) 221.

[4] P. S. Aspinwall and C. A. Lütken, "Quantum Algebraic Geometry of Superstring Compactifications," Nucl. Phys. B355 (1991) 482.

[5] P. S. Aspinwall and D. R. Morrison, "U-Duality and Integral Structures," hep-th 9505025, Phys. Lett. 355B (1995) 141.

[6] K. Becker, M. Becker and A. Strominger, "Fivebranes, Membranes and Nonperturbative String Theory," hep-th 9507158, Nucl. Phys. B456 (1995) 130.

[7] N. Berkovits and C. Vafa, "N=4 Topological Strings," hep-th 9407190, Nucl. Phys. B433 (1995) 123.

[8] D. Joyce, "Compact 8-Manifolds with Holonomy Spin(7)," Invent. Math. 123 (1996) 507.

[9] H. Ooguri, Y. Oz and Z. Yin, work in progress.

[10] N. Ishibashi, "The Boundary and Crosscap States in Conformal Field Theories," Mod. Phys. Lett. A4 (1989) 251.

[11] A. Strominger, "Special Geometry," Comm. Math. Phys. 133 (1990) 163.

[12] P. Candelas and X. C. de la Ossa, "Moduli Space of Calabi-Yau Manifolds," Nucl. Phys. B355 (1991) 455.

[13] S. Cecotti and C. Vafa, "Topological Antitopological Fusion," Nucl. Phys. B367 (1991) 359.

[14] M. Bershadsky, S. Cecotti, H. Ooguri and C. Vafa, "Kodaira-Spencer Theory of Gravity and Exact Results for Quantum String Amplitudes," hep-th 9309140, Comm. Math. Phys. 165 (1994) 311.

[15] B. R. Greene, D. R. Morrison and M. R. Plesser, "Mirror Manifolds in Higher Dimension," hep-th 9402119, Comm. Math. Phys. 173 (1995) 559.

[16] B. R. Greene and M. R. Plesser, "Duality in Calabi-Yau Moduli Space," Nucl. Phys. B338 (1990) 15.

[17] D. R. Morrison, "Where is the Large Radius Limit?," hep-th 9311049, Proceedings of Strings 93. 
[18] P. Candelas, X. C. De La Ossa, P. S. Green and P. Parkes, "A Pair of Calabi-Yau Manifolds as an Exactly Solubule Superconformal Theory,” Nucl. Phys. B359 (1991) 21.

[19] D. Morrison, "Mirror Symmetry and Rational Curves on Quintic Threefolds: A Guide for Mathematicians," J. Amer. Math. Soc. 6 (1993) 223.

[20] W. Schmid, "Variation of Hodge Structure: The Singularities of the Period Mapping," Invent. Math. 22 (1973) 211.

[21] D. R. Morrison, "Making Enumerative Predictions by Means of Mirror Symmetry," alggeom 9504013, Essays on Mirror Manifolds II.

[22] A. Strominger, S.-T. Yau and E. Zaslow, "Mirror Symmetry is T-Duality," hep-th 9606040.

[23] D. R. Morrison, "Mirror Symmetry and the Type II String," hep-th 9512016.

[24] E. Witten, "Bound States of Strings and p-Branes," hep-th 9510135, Nucl. Phys. B460 (1996) 335.

[25] M. R. Douglas, "Branes within Branes," hep-th 9512077.

[26] C. Vafa and E. Witten, "On Orbifolds with Discrete Torsion," hep-th 9409188, J. Geom. Phys. 15 (1995) 189.

[27] C. Vafa, "Instantons on D-branes," hep-th 9512078, Nucl. Phys. B463 (1996) 435.

[28] M. R. Douglas and M. Li, "D-Brane Realization of $N=2$ Super Yang-Mills Theory in Four Dimensions," hep-th 9604041.

[29] D. Gepner, "Exactly Solvable String Compactifications on Manifolds of $S U(N)$ Holonomy," Phys. Lett. 199B (1987) 380. 